growth and development. There were 169 female twin pairs, 161 male twin pairs, and 6o opposite-sex pairs. Age trends showed an appreciable darkening in both hair and eye color for both sexes over age. There was, however, a consistent sex difference with more males having lighter-color hair and eyes than females. In addition, the color for males was more likely to remain stable from age to age, whereas the color for females was more likely to change.

Within-pair concordance for color was calculated for all same-sex twins whose zygosity had been determined through blood typing. Despite the general change in both hair and eye color, the $\mathrm{MZ}$ twins were significantly more concordant than $\mathrm{DZ}$ twins at every age, indicating a strong genetic influence on the timing of color changes. Results are discussed in terms of accelerated maturation of females, and the need for genetic models, which are both age- and sex-specific, as far as hair and eye color are concerned.

Ronald S. Wilson, Ph.D., Medical-Dental Research Bldg., Rm. I I H, P.O. Box I055, Louisville, Kentucky $4020 \mathrm{r}$, USA

\section{CHRONOGENETIC MEANING OF FETAL MEMBRANES IN TWINNING}

\section{GEDDA, G. BRENGI, R. GAZORA}

The Gregor Mendel Institute of Medical Genetics and Twin Research, Rome, Italy

The ovular annexes in $M Z$ twin pregnancies seem to be particularly important to assess the times of gemellogenesis, i.e., the moment at which the zygote, or the early embryo, give origin to the separate ontogenesis of the two or more members of an MZ twinning.

A research has been carried out in the obstetrical departments of two important hos- pitals of Rome. The full chronogenetic significance of this research will become clear once it is possible to verify that the times of the $\mathrm{MZ}$ gemellogenesis are in turn controlled by heredity.

As for the ovular annexes of polyzygotic pregnancies, these may be used to assess the hereditary variability of the early ontogenetic times with respect to single births, the coeteris paribus condition being realized, here too, by the simultaneous character of fertilization and pregnancy.

Prof. Luigi Gedda, Istituto Mendel, Piazza Galeno 5, oor6i Roma, Italy

\section{FERTILITY AND LIFE SPAN IN DROSOPHILA MELANOGASTER AGCORDING TO MATERNAL AGE}

\author{
L. GEDDA, G. BRENCI
}

The Gregor Mendel Institute of Medical Genetics and Twin Research, Rome, Italy

Fertility and average life span in relation to maternal age have been studied in the Drosophila melanogaster strains: Oregon $r$; $C n, b w, v g$; and in the two hybrid strains, (male) Oregon $r \times C n$, bw, vg (female), and (male) $C n$, bw, vg $\times$ Oregon $r$ (female).

For each crossing, the female offsprings have been studied, obtained from matings limited to one single day, i.e., the eighth day of opening, for males, and to the fourth, eighth, and twelfth day for females, i.e., at the beginning, the middle, and the end of the fertile period. In order to limit random variability, the crossings were repeated five times. For each experimental block, the number of births and the life span of the female offsprings were considered. A marked covariability is shown to exist between maternal age and both fertility and life span.

Prof. Luigi Gedda, Istituto Mendel, Piazza Galeno 5, oor6I Roma, Italy 